\title{
sciendo
}

\section{Health Technology Assessment: The Role of Total Cost of Ownership}

\author{
Eoghan McConalogue, Paul Davis, Regina Connolly \\ Dublin City University, Dublin, Ireland
}

\section{Abstract}

Background: Recently the European Union, through its Horizon 2020 fund has awarded numerous research projects tasked with the development of cloud-based health technology solutions. A number of these projects have a specific focus on ambient assisted living ( $A A L$ ) technologies; solutions that offer a unique opportunity to improve the quality of life of persons with mild cognitive impairments. The diffusion of these solutions across a European wide cloud infrastructure presents a novel opportunity to reduce economic pressures currently experienced by European health systems. However, no route to market framework currently exists for a European wide healthcare delivery system. Objectives: The goal of the paper is to conduct the review and develop the literature around technology assessment for AAL technologies and route to market frameworks. Methods/Approach: We highlight the role of Total Cost of Ownership (TCO) when conducting a technology assessment by reviewing existent literature. Results: We discuss three TCO models that can support the assessment of AAL technologies. Conclusions: There is a gap in the analysis of TCO models in the context of AAL technologies particularly in public and private sector collaborations. TCO process should be developed into a key award criterion when conducting AAL technology assessment and procurements, thus aiding long term strategic decision making.

Keywords: technology assessment, ambient assisted living, total cost of ownership, health systems, procurement

JEL classification: I1 1

Paper type: Research article

Received: Dec 10, 2017

Accepted: Feb 20, 2019

Citation: McConalogue, E., Davis, P., Connolly, R., (2019), "Health Technology Assessment: The Role of Total Cost of Ownership", Business Systems Research, Vol. 10 No. 1, pp.180-187.

DOI: $10.2478 / \mathrm{bsrj}-2019-0013$

\section{Introduction}

World age profiles are changing. Recent research publications suggest the most extreme population changes are to be expected in western Europe and northern Asia, specifically Japan. Immediate changes are more apparent in the Japanese population where almost one quarter of the population is already over the age of 60 . It is predicted that almost half of the Japanese population will be over the age of 60 the year 2060. While the projected population changes are not as severe in Europe it is nonetheless predicted that people over the age of 65 will outnumber people under 
this age 2 to 1 by 2060 (Eurostat, 2011; SBJ, 2011). While in 2011 there were 535,393 people over the age of 65 in Ireland, a 14\% increase from 2005 (Central Statistics Office, 2012).

In tandem with a change in world age profiles has been the development of ambient assisted technologies (AAL), (Novitzky et al., 2015; Jacquemard et al., 2014). AAL technologies attempt to utilise sensory and cloud technologies as an eHealth solution. It is envisioned that these solutions will provide assistance and support to persons suffering various diseases such as cardio vascular disease (CVD) and mild cognitive impairment (MCl). Two horizon 2020 projects are currently tasked with the development of AAL technologies. These projects are timely, as age profiles develop so too will age related diseases. It is therefore imperative that efforts are made to research and develop technologies that can improve the expected quality of life (QOL) of persons suffering with $\mathrm{MCl}$ and such diseases. Moreover, the inevitable pressure that health care systems will feel require a degree of scenario planning and research that might lessen this pressure in the future (Novitzky et al., 2015).

The promise of economic advantage and relief of health systems cannot occur unless appropriate market assessment tools for of AAL technologies are developed. The procurement and assessment of AAL technologies is a complex multi-layered process, particularly in the context of fragmented European health systems. Although technology assessment itself is not a new concept, it has a documented history spanning over 60 years, its application in the present context is somewhat new and undocumented. The fundamentals of a technology assessment however remain the same. It must consider cost, effectiveness and readiness of the technology for a given market. In doing so all stakeholders, bearers of costs and market alternatives should be reviewed (National Library of Medicine, 2004; O'Donnell et al., 2009; National Institute for Health Research, 2017). It is a multidisciplinary field. In this present context it includes technological analysis across medical, social, ethical and economic arenas of development. While policy relating to diffusion and use of health technology must also be considered (INAHTA, 2017).

Work carried out in other jurisdictions such as the UK was reviewed as part of the background for adopting an approach to impact assessment for the AAL supports. Although there was anecdotal evidence that procurement was being involved in the HTA in the UK, when the implementation documents were reviewed there was only one reference to purchasing in the NHS "Guide to the multiple technology appraisal process" published in October 2009.

In Ireland for example HTA is governed by HIQA (Health Information \& Quality Authority, 2010). Similar to the UK documentation, in all of HIQA's 3 recent publications the role of procurement in helping to examine the cost benefits or the economic evaluation is not mentioned (Health Information \& Quality Authority, 2017). Procurement as such is not seen as having a role to play in the engagement with suppliers of medical devices, rather the role falls to clinicians and specialist staff. This lack of linkage between the early involvement of procurement and the development of innovation goes counter to previous government recommendations for the procurement of innovation. This approach may indicate a lack of consistency between government policy and actual implementation.

This work offers insight into the challenges that any Pan-European project with a healthcare delivery focus will be faced with. After reviewing various health systems and considering that the history of Europe begins together different forms of healthcare governance from Bismarck models to more centralised models, it becomes clear that integration of a 'one size fits all' model is almost impossible to create (World Health Organisation, 2016). Extant research (Kidholm et al., 2012; 
Janssen et al., 2013; Cusack et al., 2009) does show that technology assessments can coincide with route to market solutions, this research is however void of cases where such a disparate model as is found in Europe, has been catered for. As such the present study only serves to highlight the need for bespoke models and further research in this present context.

The Horizon 2020 projects referred to in this paper are being undertaken at an opportune time. Both projects require the bespoke development of total cost of ownership tools. At project maturity both solutions will have trialled suitable total cost of owner ship tools and commercialisation routes. This knowledge, in conjunction with a review of various European health systems it has made it clear that developing a bespoke total cost of ownership tool for European-wide AAL technological solutions is imperative to their successful diffusion through health systems. This paper will now present the underlying role and importance of a total cost of ownership analysis in an eHealth context.

\section{Methodology}

Due to time restrictions and the nascence of research in the area of TCO models being applied to AAL technology assessment a secondary research approach was employed for this paper (Bryman and Bell, 2007). A literature review of extant TCO models and their application to AAL was performed with the models applicable to eHealth solutions then presented in the paper.

A traditional review of literature on small and medium-sized enterprises (SMEs), public procurement processes and related government policies was undertaken. As the direction of the research was undetermined at project initiation a traditional scoping review methodology was employed as advised by Jesson, Matheson and Lacey (2011). In keeping with the method, the previous experiences of the researcher were called on to develop a base. Highly cited papers by contributors in leading academic journals were consulted. In addition, global organisations such as the Organisation for Economic Co-operation and Development (OECD) were also examined for outputs relating to key search terms and themes.

\section{Results}

The World Health Organisation states that health technology assessment involves a systematic approach to the evaluation of properties, effects and impacts of a technology. It is a multidisciplinary task that incorporates social, economic, organisational and ethical considerations with an objective to inform policy decision making (World Health Organisation, 2017). Insights from this research paper indicate that there is an alarming lack of alignment between the Health Technology Assessment (HTA) process and the overall procurement process. Where a normal technology assessment is required to begin with a review of similar historical case examples it was found that no such practice is mirrored when medical devices are being assessed. In addition, where a traditional technology assessment would include the consultation of various stakeholders, health technology assessments failed to include key stakeholders such as biomedical staff.

The dichotomy of European health systems, between public and private systems of delivery, elevates the role and influence of the assessment process relating to eHealth services. As assessors must evaluate the costs associated with the procurement of eHealth solutions during the technology assessment process, a clear understanding must be obtained of where costs gather along a product lifecycle; on the public or private side for example. 
A big picture understanding of product or service life cycles is therefore required to complete a technology assessment. Models that assess the life cycle of a technology must reflect the particular industry and body of stakeholders for which it is intended.

The procurement process is not always fully documented, particularly in the public sector. In an effort to harmonise methodologies Caldwell et al. (2007) has outlined a procurement process based on Van Weeles (2004) model. The result is a six-stage process; specification, supplier selection, contracting, ordering, expediting and follow-up / evaluation.

The assessment and procurement of AAL technologies is particularly complex. Consideration must be given to existing medical support infrastructure, the supply of utility services such as electricity, cloud services, staff training and implementation. In some institutions the assessment and procurement of AAL technologies can be perceived as a strategic activity, necessitating a medium to long-term scope. Aiding this process the Total Cost of Ownership tool (TCO) (Hurkens and Wynstra, 2006) is used to understand indirect costs. This allows organisations to assess the lowest possible cost to be incurred when in negotiation with suppliers (Van Weele, 2004).

Ellram and Siferd (1998) have identified three segments to successful TCO analysis; operational, tactical and strategic. Through the TCO analysis organisations can uncover opportunities to either avoid or reduce cost. Due to the fragmented nature of European health systems this can prove to be a difficult task. Not only is there a lack of a common framework for deployment but cost structures vary from state to state.

TCO analysis highlights the many layers involved in the assessment of a technology. For example, it is often the case that the assessment and acquisition of an AAL technology includes the considered procurement of a product and service. As a result, performing TCO analysis during a technology assessment not only informs matters relating to direct and indirect cost (Leenders et al., 2006) but also elucidates a number of other influential factors: (i) Cost reduction opportunities; (ii) Supplier evaluation and selection criteria; (iii) Data for negotiations; (iv) Points to focus suppliers on cost reduction opportunities; ( $v$ ) Advantages of expensive, high quality items; (vi) Clarification and definition of supplier performance expectations; (vii) A long term supply perspective, and (viii) Forecasting for future performance.

There are a number of methods for estimating TCO. Organisations generally choose a TCO approach from one of two overriding methodologies; a standardised TCO tool approach or the development of a bespoke tool.

Hurkens and Wynstra (2006) highlight a number of methods to use when estimating costs. The first of these, the monetary based method, which allocates the costs of purchasing a service or product to the different true costs of components in the offering.

The second method is the cost-ratio or value-based method (Carr and Ittner, 1992; Ellram, 1995). This method incorporates the monetary method with qualitative performance data. By evaluating non-monetary data, a suppler rating score can be amassed, resulting in a total cost factor (Hurkens and Wynstra, 2006).

Shin and Benton (2007) offer a third model that introduces five performance factors; quality, delivery, technology, price and service. Suppliers are given a numeric rating, the highest being 1.0, indicating hidden cost of ownership.

Although there has been considerable research carried out on life cycle costing and total cost of ownership in other jurisdictions and within other product categories, there is little evidence of this being done on AAL supports. There is scope here for further research and collaboration between the public sector and industry to develop these models. 


\section{Discussion}

This paper makes important contributions in three areas. The main finding of this paper is the existence of three TCO models that can support the assessment of AAL technologies (Hurkens and Wynstra, 2006; Carr and Ittner, 1992; Ellram, 1995; Shin and Benton, 2007). In addition, this research reveals strategic roles for TCO models that go beyond their initial purpose; providing data for negotiations, identification of cost reduction opportunities, supplier performance expectations, supplier selection criteria, advantages of expensive, high quality items and forecasting for future performance. Finally, it was found that existing models do not incorporate adequate collaboration between public and private entities.

A limitation of this paper is the lack of extant literature relating to TCO models and health technology assessment. Furthermore, there are limitations in the methodology employed; future studies should consider qualitative interviews with public and private practitioners.

The development and adoption of AAL technologies and health ICTs has grown exponentially in recent years (Angst and Agarwal, 2009; Statista, 2015; Glynn et al., 2015). While extant scientific evidence is limited there are many positive purposes for which AAL technologies could be utilised for; they can alleviate challenges related to logistics for on-site visits by patient, clinician and care giver; they can facilitate rehabilitation sessions from the comfort of a patient's home; they can reduce the economic burden that currently rests on stressed health care systems, caregivers and patients (Frontera et al., 2017).

The inherent benefit of these various technological developments is evident, despite this, barriers to use exist still exist (Bittner et al., 2015). Integration of multiple disciplines and technological solutions is still an issue in many health systems. Patients and citizens have expressed concerns over data ownership, confidentiality, privacy and security (Smith, Milberg and Burke, 1996; D'Arcy, Hovav and Galletta, 2009; Hong and Thong, 2013), while key stakeholders need a clear understanding of all underlying business commitments. These systems depend on multiple stakeholders having a clear and elucidated understanding of the technologies being developed and their infrastructure requirements. Essentially the success of these platforms is dependent on their technological acceptance and business case adoption (Harris et al., 2011). Therefore, it is imperative for researchers, practitioners and policy makers to have tools that assist in analysing the various business elements of these technologies in order to drive their adoption.

This paper has highlighted existing TCO models and the role TCO has in the development of a route to market framework for European wide healthcare delivery systems. As the proliferation of AAL technologies and cloud computing infrastructure continues, so too will the number of private and public collaborations. Healthcare systems across Europe display a diverse network of public and private partnerships; these include but are not limited to technological solutions, health care delivery systems and insurance packages. The revelation of existing TCO models, their strategic utility and the obvious room for public and private collaboration on TCO modelling should signal all stakeholders to embrace impending enterprise and research opportunities. Doing so will reduce the barriers to entry that currently exist for AAL technologies and health care in general. 


\section{Conclusions}

This paper set out to explore the extant literature relating to TCO in the context of health technology assessment. In doing so it has uncovered a number of TCO models that can be readily adapted for health technology assessment. In addition, it was revealed that these TCO models can offer utility in scenarios beyond their initial purpose, that is to say they can offer valuable insight to the business negotiation process.

Although research exists on TCO models it should be noted that there is little analysis existing on its application in the context of AAL technologies to be diffused on a European-wide scale across varying health care systems. There is an opportunity for further research to develop this area particularly with regard to public and private sector collaborations. It is recommended that future TCO models be developed in conjunction with industry partners. It is also recommended that the TCO process be developed into a key award criterion when conducting AAL technology assessment and procurements, thus aiding long term strategic decision making.

In the immediate future this research carry's a salient practical implication. It forms the basis of a TCO model that will be employed across a number of EU funded health projects. The various consortia will use the TCO model to assess risks, benefits, costs, opportunities and strategies associated with their respective health technologies. It will also be used when in negotiations with suppliers. This a key finding of this paper, in that there is utility beyond the traditional TCO model usage.

Finally, future research should consider a key limitation of this paper: no public or private TCO practitioners were approached for qualitative or quantitative analysis. Deeper analysis may uncover a bespoke TCO model that varies from those discussed in this paper.

\section{References}

1. Angst, C. M., Agarwal, R. (2009), "Adoption of Electronic Health Records in the Presence of Privacy Concerns: The Elaboration Likelihood Model and Individual Persuasion", MIS Quarterly, Vol. 33, No. 2, pp. 339-370.

2. Bittner, A. K., Wykstra, S. L., Yoshinaga, P. D., Li, T. (2015), "Telerehabilitation for people with Iow vision", Cochrane Database of Systematic Reviews, No. 8.

3. Bryman, A., Bell, E. (2007), Business Research Methods, Oxford University Press.

4. Caldwell, N., Bakker, E., Read, J. (2007), "The purchasing process in public procurement", in Knight, L., Harland, C., Telgen, J, Callender, G., McKen, J. (Eds.), Public Procurement International Cases and Commentary, England, Routledge.

5. Carr, L. P., Ittner, C. D. (1992), "Measuring the cost of ownership", Journal of Cost Management, Vol. 6, No. 3, pp. 42-51.

6. Central Statistics Office (2012), Profile 2: Older and Younger, Dublin: Stationery Office.

7. Cusack, C. M., Byrne, C., Hook, J. M., McGowan, J., Poon, E. G., Zafar, A. (2009), "A Health Information Technology Evaluation Toolkit: 2009 Update", AHRQ Publication, No. 9, pp. 159.

8. D'Arcy, J., Hovav, A. and Galletta, D. (2009), "User awareness of security countermeasures and its impact on information systems misuse: A deterrence approach", Information Systems Research, Vol. 20, No. 1, pp. 79-98.

9. Ellram, L. M. (1995), "Total cost of ownership: An analysis approach for purchasing", International Journal of Physical Distribution and Logistics, Vol. 25, No. 8, pp. 4-23.

10. Ellram, L. M., Siferd, S. P. (1998), "Total Cost of Ownership: A key concept in Strategic Cost Management Decisions", Journal of Business Logistics, Vol. 19, No. 1, pp. 55-84.

11. Eurostat (2011), "Population projections", European Commission, available at: http://epp.eurostat.ec.europa.eu/statistics explained/index.php/Population projections (7 June 2017). 
12. Frontera, W. R., Bean, J. F, Damiano, D., Ehrlich-Jones, L., Fried-Oken, M., Jette, A., Jung, R., Lieber, R. L., Malec, J. F., Mueller, M. J., Ottenbacher, K. J., Tansey, K. E., Thompson, A. (2017), "Rehabilitation Research at the National Institutes of Health: Moving the Field Forward (Executive Summary)", The American Journal of Occupational Therapy, Vol. 71, No. 3, pp. $1-12$.

13. Glynn, L. Casey, M., Walsh, J., Hayes, P. S., Harte, R. P., Heaney, D. (2015), "Patients' views and experiences of technology based self-management tools for the treatment of hypertension in the community: A qualitative study", BMC Family Practice, Vol. 16, No. 1.

14. Health Information \& Quality Authority (2017), "HIQA publishes guide to health technology assessment", available at: https://www.hiqa.ie/hiqa-news-updates/hiqa-publishes-guidehealth-technology-assessment (25 November 2017).

15. Health Information \& Quality Authority (2010), "Guidelines for the Economic Evaluation of Health Technologies in Ireland", available at: https://www.ispor.org/peguidelines/source//reland_Economic_Guidelines_2010.pdf (25 November 2017).

16. Harris, P. R., Sillence, E., Briggs, P. (2011), "Perceived Threat and Corroboration: Key Factors That Improve a Predicitive Model of Trust in Internet-based Health Information and Advice", Journal of Medical Internet Research, Vol. 13, No. 3.

17. Hong, W., Thong, J. Y. L. (2013), "Internet privacy concerns: An integrated conceptualization and four empirical studies", MIS Quarterly, Vol. 37, No. 1, pp. 275-298.

18. Hurkens, K., Wynstra, F, (2006), "The concept "Total Value of Ownership": A case study approach", available at: www.iimm.org/knowledge_bank/IFPSM (7 June 2017).

19. INAHTA (2017), "Health Technology Assessment", available at: http://www.inahta.org/hta (25 November 17)

20. Jacquemard, T., Novitzky, P., O'Brolcháin, F., Smeaton, A. F., Gordijn, B. (2014), "Challenges and opportunities of lifelog technologies: a literature review and critical analysis", Science and Engineering Ethics, Vol. 20, No. 2, pp. 379-409.

21. Janssen, R., Hettinga, M., Prins, H., Visser, S., Mengo, R., Krediet, I., Haaker, T., Bodenstaff, L. (2013), "Developing evidence guidelines for eHealth small and medium-sized enterprises", in Proceedings of the Fifth International Conference on eHealth, Telemedicine, and Social Medicine (eTELEMED), pp. 92-95.

22. Jesson, J., Matheson, L., Lacey, F. M. (2011), Doing Your Literature Review: Traditional and Systematic Techniques, SAGE Publications.

23. Kidholm, K., Ekeland, A. G., Jensen, L. K., Rasmussen, J., Pedersen, C. D., Bowes, A., Flottorp. S. A., Bech, M. (2012), "Model for Assessment of Telemedicine Applications: Mast", International Journal of Technology Assessment in Health Care, Vol. 28, No. 1, pp. 44-51.

24. Leenders, M., Johnson, F., Flynn, A., Fearon, H. (2006), Purchasing and Supply Management, 13th edition, New York, Irwin McGraw Hill.

25. National Institute for Health Research (2017), "Health Technology Assessment", available at: $\quad$ https://www.nihr.ac.uk/funding-and-support/funding-for-research-studies/fundingprogrammes/health-technology-assessment/ (25 November 2017).

26. National Library of Medicine (2004), "HTA 101: Introduction to Health Technology Assessment", available at: https://www.nlm.nih.gov/nichsr/hta101/ta10103.html

(25 November 2017).

27. Novitzky, P., Smeaton, A. F., Chen, C., Irving, K., Jacquemard, T., O'Brolcháin, F., O'Mathúna, D., Gordijn, B. (2015), "A Review of Contemporary Work on the Ethics of Ambient Assisted Living Technologies for People with Dementia", Science and Engineering Ethics, Vol. 21, No. 3, pp. 707-765.

28. O'Donnell, J. C., Pham, S. V., Pashos, C. L., Miller, D. W., Smith, M. D. (2009), "Health Technology Assessment: Lessons Learned from Around the World-An Overview", Value in Health, Vol. 12, No. 2, pp. S1-S5.

29. SBJ (2011), "Statistical handbook of Japan 2011 ", Statistics Bureau and the Director-General for Policy Planning of Japan, available at: http://www.stat.go.jp/english/data/handbook/index.htm (7 June 2017).

30. Shin, H., Benton, W. C. (2007), "A quantity discount approach to supply chain coordination", European Journal of Operational Research, Vol. 180, No. 2, pp. 601-616. 
31. Smith, H. J., Milberg, S. J., Burke, S. J. (1996), "Information Privacy: Measuring Individuals' Concerns about Organizational Practices", MIS Quarterly, Vol. 20, No. 2, pp. 167-196.

32. Statista (2015), "Statistics and facts about mobile app usage", available at: http://www.statista.com/topics/1002/mobile-app-usage (22 June 2016).

33. Van Weele, A. J. (2004), Purchasing and Supply Chain Management, 4th edition, Cengage Learning Business Press.

34. World Health Organisation (2016), "From Innovation to Implementation: eHealth in the WHO European Region", available at: http://www.euro.who.int/en/publications/abstracts/frominnovation-to-implementation-ehealth-in-the-who-european-region-2016 (7 June 2017).

35. World Health Organisation (2017), "Health technology assessment", available at: http://www.who.int/medical_devices/assessment/en (7 June 2017).

\section{About the authors}

Eoghan McConalogue is a PhD candidate and Assistant Professor at Dublin City University. His research has a focus on eHealth technologies, self-disclosure and trust. Author can be contacted at eoghan.mcconalogue@dcu.ie.

Dr. Paul Davis is a lecturer in the School of Business at Dublin City University. He is a past council member and past president for the Irish Institute of Purchasing and Materials Management. He has previously been seconded to the Health Service Executive ICT Strategy Unit, advising on procurement strategy and market engagement. Dr Davis was lead PI in DCU for the Winning in Tendering Project. The project has a budget of $€ 3.7$ million euro. He is currently $\mathrm{PI}$ for the following $\mathrm{H} 2020$ projects in DCU Business School: Pathway, Magic, Inlife. The author can be contacted at paul.davis@dcu.ie.

Prof Regina Connolly has significant expertise in healthcare technology impact assessment as well as eHealth business model development and is a Lead Investigator in several international Ambient Healthcare Technology research projects that were awarded over $€ 8.43$ million in funding from the European Commission in 2014. She is also a lead investigator in a pre-commercial health technology procurement proposal that was awarded over $€ 5.1$ million in European funding in 2015. In 2016, the H2O2O public health research project (MIDAS) which she co-leads with Ulster University was awarded $€ 3.1$ million in European funding. The author can be contacted at regina.connolly@dcu.ie. 